\title{
Extending Network Life by Using Mobile Actors in Cluster-based Wireless Sensor and Actor Networks
}

\author{
Nauman Aslam, William Phillips, William Robertson and S. Sivakumar \\ Department of Engineering Mathematics \& Internetworking \\ Dalhousie University, Halifax, Nova Scotia, Canada, B3J2X4 \\ \{naslam, william.phillips, bill.robertson,sivas\}@dal.ca
}

\begin{abstract}
Wireless sensor actor networks (WSANs) consist of a large number of resourceconstrained nodes (sensors) and a small number of powerful resource rich nodes (actors). This paper investigates the case where sensors are organized into clusters and mobile actors are used for maintaining an energy efficient topology by periodically manipulating their geographical position. We present an elegant technique that allows actor nodes to find an optimal geographical location with respect to their associated cluster heads such that the overall energy consumption is minimized. The simulation results demonstrate that the technique proposed in this paper significantly minimizes energy consumption and extends the network lifetime compared with traditional cluster-based WSN deployments.
\end{abstract}

Keywords: wireless sensor actor networks, mobile actors, energy conservation, clustering, network lifetime.

\section{Introduction}

Wireless Sensor Actor Networks (WSANs) have emerged as the-state-of-the-art technology in data gathering from remote locations by interacting with physical phenomena and relying on collaborative efforts by few resource rich and a large number of low cost devices [1]. In WSANs sensors are low cost resource constrained devices that have limited energy, communication and computation capabilities. Once deployed, sensor nodes collect the information of interest from their on board sensors, perform local processing of these data including quantization and compression, and forward the data to a base station (BS) through a cluster head or gateway node. Actors, on the other hand are resource rich nodes that are equipped with better possessing power, higher transmission powers, more energy resources and may contain additional capabilities such as mobility.

Despite the tremendous technological advancements in the field of wireless sensor network (WSNs), energy conservation is still one of the fundamental challenges in WSN system design. In a sensor node, energy is consumed in sensing, computation and processing, however the wireless transceiver consumes a significant amount of energy as compared to all other sources. Most of the WSN deployments are based on

Please use the following format when citing this chapter:

Aslam, N., et al., 2008, in IFIP International Federation for Information Processing, Volume 264; Wireless Sensor and Actor Networks II; Ali Miri; (Boston: Springer), pp. 74-84. 
an application specific data gathering i.e. a large number of sensor nodes send their data to the BS. Clustering is employed as a standard approach for achieving energy efficiency and scalable performance in WSNs [2]. Sensor nodes can be organized hierarchically by grouping them into clusters, where the data is collected and processed locally at the cluster head. Single hop clustering protocols follow two-tier architecture, consisting of sensor nodes and cluster heads. In tier one communication, each sensor node sends it data to its local cluster head. In tier two the cluster heads send the processed data to the BS. In protocols where the cluster head selection is performed randomly, the distribution of cluster heads is not well controlled. Most protocols based on the two-tier architecture use direct communication for sending cluster head data to the BS. If a cluster head is located far away from the sink, its energy consumption would be greater than the one located closer to the sink for similar traffic patterns from cluster members. Intuitively, deployment of resource rich actor nodes could off set some of the higher transmission costs incurred by cluster heads in data transmission to the BS. In such a case, each elected cluster head will associate with an actor node which will forward cluster head's data to the BS. Since random clustering involves election of new cluster heads in each round therefore deployment of static actor nodes will not be energy efficient. Such inefficiency may result from variations in distances between the actor node and its associated cluster heads and their respective residual energy levels. The concept of mobility solves this dilemma by effective location management of actor nodes thus providing the ability for enhanced energy savings. Figure 1 illustrates a scenario where mobile actors are used for relaying cluster head's data to the BS. This paper proposes a methodology where mobile actor nodes relocate to a new location in each round such that the energy consumed by associated cluster heads is minimized. A weighted cost based function that accounts for the residual energy levels of associated cluster heads is used to find the new of location the actor nodes.

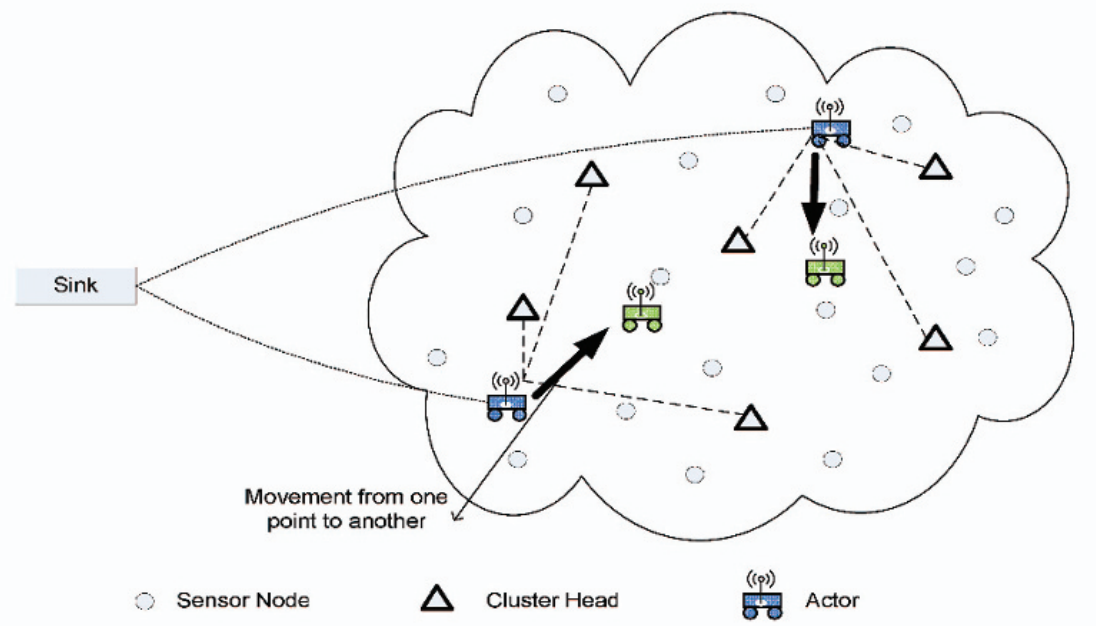

Figure 1: Example of Sensor Field with Cluster Heads and Mobile Actors 
The concept of mobile nodes in WSN is relatively new and has been proposed in various contexts. One of the earliest proposed researches was concept of data mules [3]. Data mules are referred as mobile entities that collect data from sensors as they pass by the sensor nodes buffer the collected data and drop the data off to the wired access point. Data mules were primarily aimed at collection of non real-time data. Authors in [4] used mobile nodes for data collection in sparsely connected network. First nodes are partitioned into several groups with respect to their data generation rates and location. Then the schedule for each group is created using the traveling salesman problem such that there is no data loss or overflow. Message Ferrying is introduced in $[5,6]$ where a set mobile nodes (message ferries) provide communication service for nodes in sparse connected networks. In the message ferry approach, each node communicates only with the message ferries, therefore all communication cost is reduced to a short distance single hop communication at the expense of increased message delay. This paper adopts a rather simple approach by exploiting the actor mobility to achieve balanced energy dissipation by cluster heads. The rest of the paper is organized as follows. Section 2 presents system model and assumptions. Section 3 presents details of our proposed technique for location management of mobile actors. Simulation results are discussed in Section 4. Finally, our main conclusions and future research directions are highlighted in Section 5.

\section{System Model and Assumptions}

We make the following assumption for our sensor network:

1. The network consists of a large number of resource constrained nodes (referred as sensing nodes) and small number of resource rich nodes (referred as actor nodes) with enhanced communication capacity, ability to recharge their energy source and mobility.

2. We assume that all nodes have means to find out their geographical position.

3. The nodes are dispersed in a 2-dimensional space following a uniform distribution.

4. The base station is located outside the deployment region and has no energy constraints.

5. Once deployed the sensor nodes remain stationary and continue to operate until they completely exhaust their batteries.

6. We use the energy model presented in [7]. In this model the energy required to run the transmitter or receiver electronic circuitry $E_{\text {elct }}$ is $50 \mathrm{pJ} / \mathrm{bit}$ and $\varepsilon_{a m p}$ the energy to run transmitter amplifier is $100 \mathrm{pJ} / \mathrm{bit} / \mathrm{m}^{2}$. The energy expended by a node in transmitting an $l$-bit message a distance of $\mathrm{d}$ is given by,

$$
E_{T x}=l E_{\text {elect }}+l \varepsilon_{\text {amp }} d^{2}
$$

The energy expended in receiving an $l$-bit message is given by,

$$
E_{R x}=l E_{\text {elect }}
$$




\section{Location Management of Mobile Actors}

In this section we present our rationale and algorithm for location management of mobile actors. The presented technique is based on the motivation that all cluster heads should have a balanced energy dissipation rate. This objective is achieved by introducing a cost function that allows the actor nodes to adjust their geographical location in such a manner that the overall energy of associated cluster heads is minimized. To further elaborate, let's consider an example illustrated in Figure 2. Four cluster head $A, B, C$ and $D$ are associated with an actor node. The location of each node is shown below in ' $x$ ' and ' $y$ ' coordinates. Figure $2 \mathrm{a}$ shows the initial location of actor node and its associated cluster heads. Since distance from each cluster clusters head to the actor node is different, it is impossible have comparable energy consumption for all cluster heads. Intuitively, finding a centroid as illustrated in Figure $2 b$ with respect all cluster head locations would provide a position where actor node is at the minimum distance from each cluster head. However, finding a centroid would still leads to uneven energy consumption rate if the residual energy levels of all associated cluster heads are different. To account for the residual energy levels we introduce a weighted cost based functions that allows actors node to fine tune its location based on the residual energy level. Figure $2 \mathrm{~b}$ also shows the actor node's updated location where it moves closer to the node $A$ that has minimum residual energy. Now we describe calculation of cost factor. Table 1 outlines the notation used in the following sections.

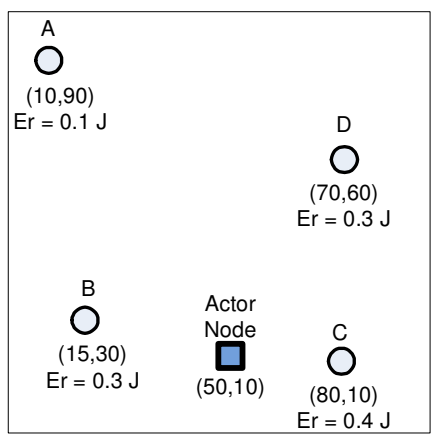

(a) Initial node locations

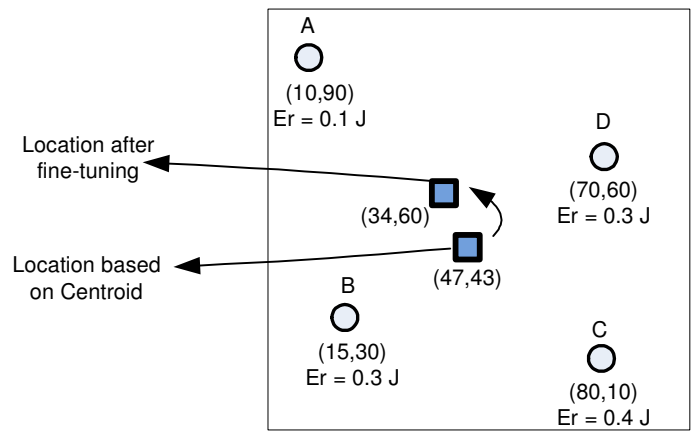

(b) Location of actor node based on cost function

Figure 2: Example illustrating location management of actor node using weighted cost function 
Table 1: Notations Used

\begin{tabular}{|l|l|}
\hline Set of actor nodes & $A$ \\
\hline$k^{\text {th }}$ node in $A$ & $a_{k}$ \\
\hline Target location coordinates of $a_{k}$ & $\left(X_{k}, Y_{k}\right)$ \\
\hline Set of cluster heads & $C H$ \\
\hline$i^{\text {th }}$ node in $C H$ & $c h_{i}$ \\
\hline Location of $i^{\text {th }}$ cluster head & $\left(x_{i}, y_{i}\right)$ \\
\hline Residual energy of $i^{\text {th }}$ cluster head & $e_{i}$ \\
\hline Cost factor for $i^{\text {th }}$ cluster head & $\varepsilon_{i}$ \\
\hline
\end{tabular}

We assume that cluster heads are elected using any generic single hop clustering protocol such as $[2,8,9]$. Once elected, each cluster head will listen for the Actor Advertisement Messages (sent by actor nodes). Each cluster head then finds a least cost actor node and sends an Association Message to the selected actor node indicating its location coordinates and residual energy level. Upon receiving the Association Message the actor node knows that it will forward cluster heads data to the BS. Once the actor node receives Association Message from all it calculates the cost factor for each cluster head $i$ as follows,

$$
\varepsilon_{i}=\frac{\left(e_{\max }-e_{i}\right)^{2}+e^{2}{ }_{\min }}{\left(e_{\max }-e_{\min }\right)^{2}+e^{2}}
$$

Where,

$$
\begin{array}{ll}
e_{\max }=\max \left(e_{i}\right) & \text { for } i \in C H^{k} \\
e_{\min }=\min \left(e_{i}\right) & \text { for } i \in C H^{k}
\end{array}
$$

The calculated cost factors are used to find the new location coordinates $X_{k}$ and $Y_{k}$ from equation (4) and (5) respectively.

$$
\begin{aligned}
& \frac{d}{d x} \sum_{i} \varepsilon_{i}\left(x-x_{i}\right)^{2}=0 \\
& \frac{d}{d y} \sum_{i} \varepsilon_{i}\left(y-y_{i}\right)^{2}=0
\end{aligned}
$$

Based on the discussion related to Figure 2 above, each mobile actor node utilizes the cost function to optimally adjust its coordinates. This is done so by taking into account the residual energy levels of its associated cluster heads and their 
communication cost to the actor node. The pseudo code of the steps used for location management in cluster based environments is outlined in Algorithm-I.

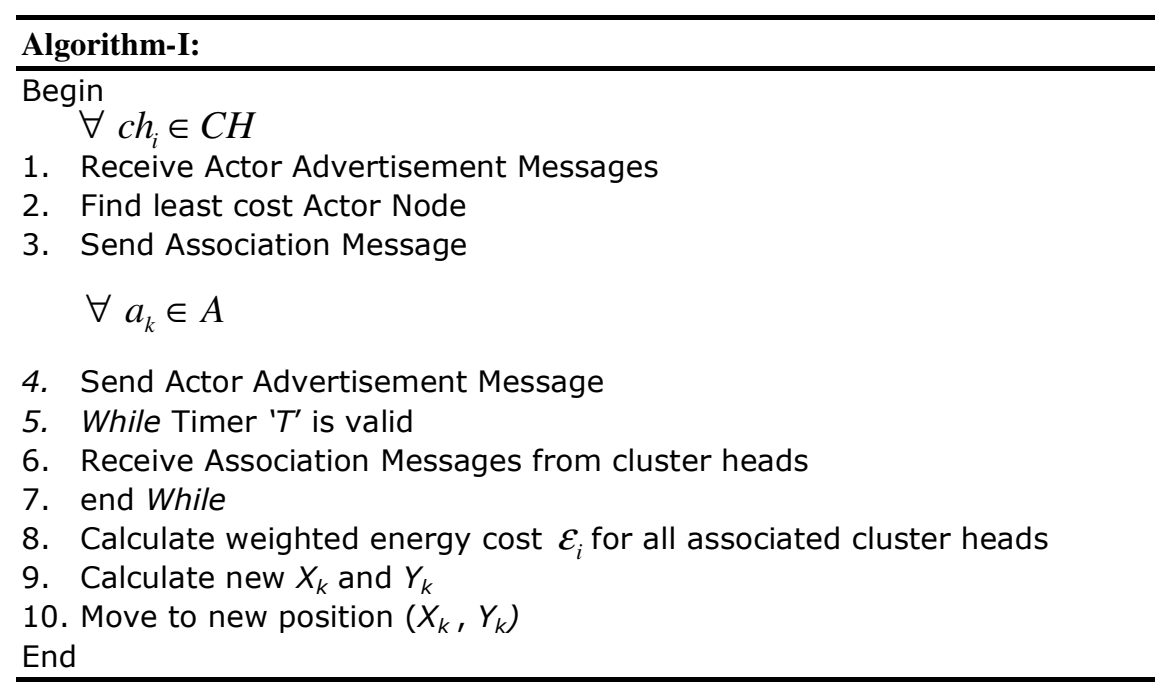

\section{Simulation Results}

This section presents the performance analysis of our proposed technique. We assume ideal conditions at the physical level such that the probability of wireless interference is negligible. The network simulation model was built using MATLAB. We used the clustering algorithm presented in [8]. We also assume that the network consists of a small number of actor nodes (ranges from $1 \%$ to $3.5 \%$ of the network size). The simulation proceeds in data collection rounds. Since each data collection round consists of cluster set up and actor node selection phase. In each round, each non cluster head node sends one data packet to its cluster head. The cluster head performs in-network processing by aggregating the data received from its member nodes and forwards this data to the actor node. Finally, the actor node forwards data received from its associated cluster head to the BS. We also assume that the actor nodes do not transmit any data while they are moving from one location to another. Mobility feature is only used to optimize the energy conservation. Two performance metrics are used. The first one is denoted by network lifetime as used in $[2,8,9]$. This metric represents network lifetime in data collection rounds from the instant the network is deployed to the moment when the first node runs out of energy. The second performance metric is the average cluster head energy consumed per round. Table 2 summarizes the important simulation parameters used.

It can be observed from Figure 3 and 4 that by using mobile actors the network lifetime is increased in more than $100 \%$ as compared to the direct transmission case. It is also worth noticing the sharp slope for mobile actor's case which is a result of balanced energy dissipation in the sensor nodes. 
Table 2: Simulation Parameters

\begin{tabular}{|l|l|}
\hline Sensor Deployment Area & $100 \times 100 \mathrm{~m}$ \\
\hline BS Location & $(50,175) \mathrm{m}$ \\
\hline Number of Nodes (N) & 200,400 \\
\hline Data Packet Size & 4000 bits \\
\hline Control Packet Size & 25 bytes \\
\hline Initial Energy of Actors & $20 \mathrm{~J}$ \\
\hline Initial Energy of Sensors & $0.5 \mathrm{~J}$ \\
\hline Number of Actor Nodes & $1 \%$ to $3.5 \%$ of N \\
\hline$E_{e l e c t}$ & $50 \mathrm{n} \mathrm{J} / \mathrm{bit}$ \\
\hline$\varepsilon_{m p}$ & $100 \mathrm{pJ} / \mathrm{bit} / \mathrm{m}^{2}$ \\
\hline
\end{tabular}

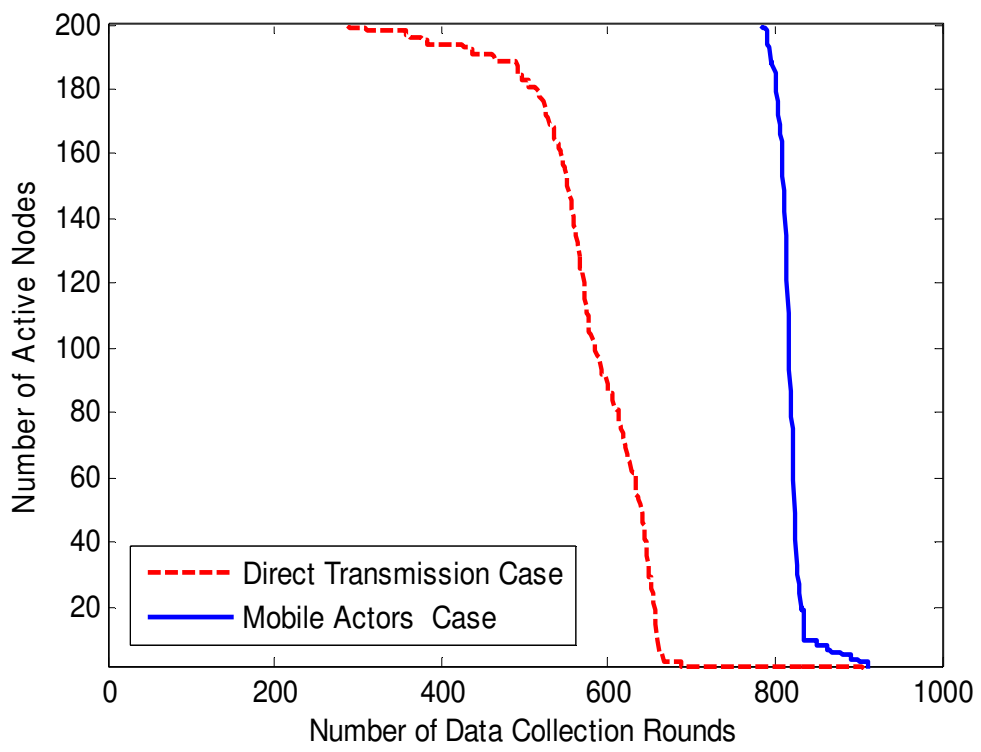

Figure 3: Network life (200 Nodes Network) 


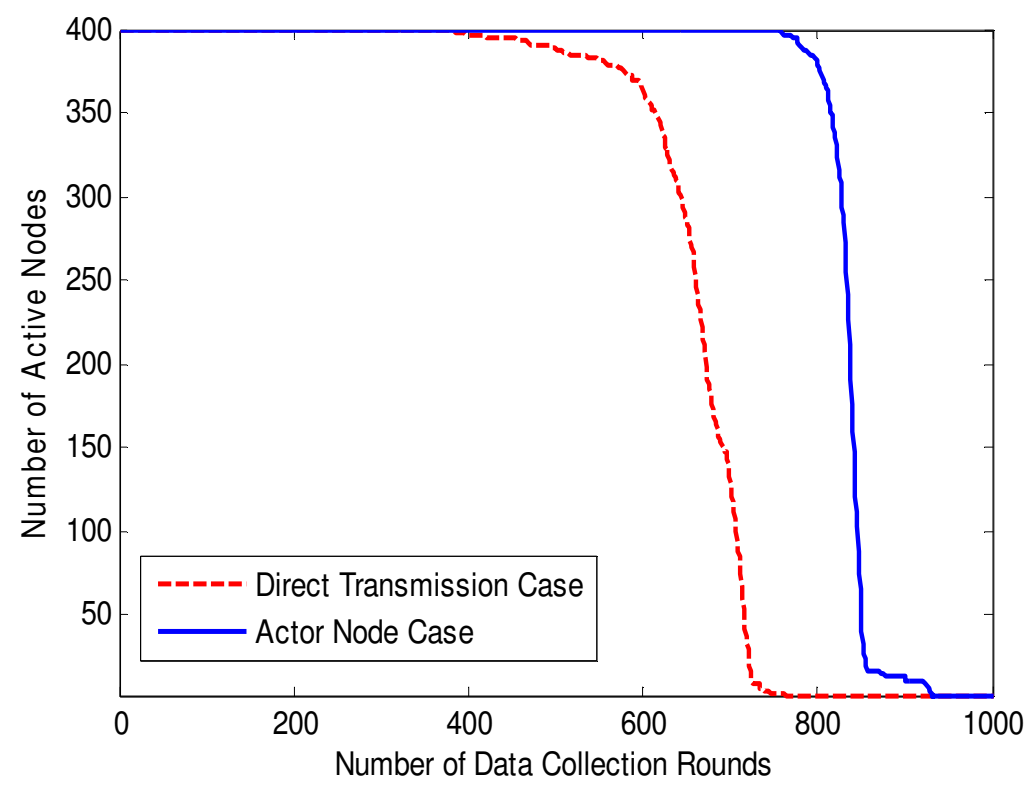

Figure 4: Network life (400 Nodes Network)

We also investigated the optimal number of actor nodes for the two network sizes of 200 and 400 nodes. Figure 5 and 6 show how network life is effected as a result of varying the number of actor nodes. The x-axis denotes the number of mobile actors in the system and the y-axis denotes the number of rounds when the first node runs out of its energy. The number of mobile actors is varied from $1 \%$ of network size to $3.5 \%$ of the network size. It can be observed from both Figure 5 and 6 that a value of $2 \%$ (4 in 200 nodes network and 8 in 400 nodes network) provides the best results. When the number of mobile actors is $1 \%$ of the network size the number of associated cluster heads per actor node is large. Hence it may lead to situations where the resultant topology cannot be further optimized. Therefore adding a small number of actor nodes further increases energy savings resulting in enhanced network lifetime. Conversely, a relatively large number of actor nodes will results in more overhead messages. Hence it is desirable to have a minimum number of actor nodes that provide optimal coverage per cluster head. From the simulations we conclude that the best value for network life time is given by when the number of mobile actors is $2 \%$ of the network size. 


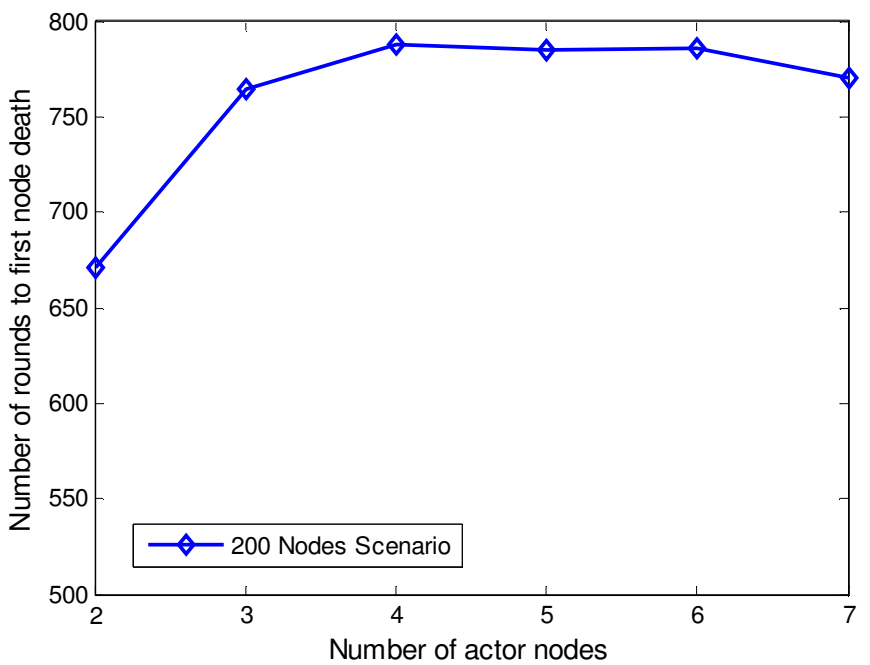

Figure 5: No. of actors vs. network life in first node death (200 Nodes Networks)

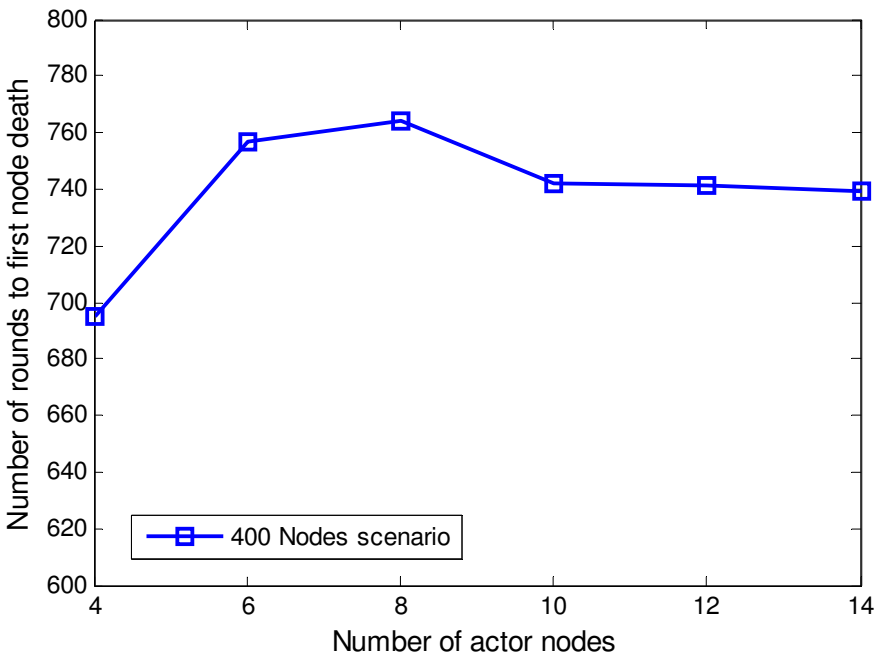

Figure 6: No. of actors vs. network life in first node death (400 Nodes Networks)

Figure 7 illustrates the mean cluster head energy consumed per round for mobile actors and direct communication scenario. The statistics are collected using 100 independent runs. It is clearly observed that the cluster heads in former case consume far less energy (almost 50\%) because of shorter distance transmission to the mobile 
actors as compare to the direct communication case. Hence the overall effect in network life time gain is quite significant.

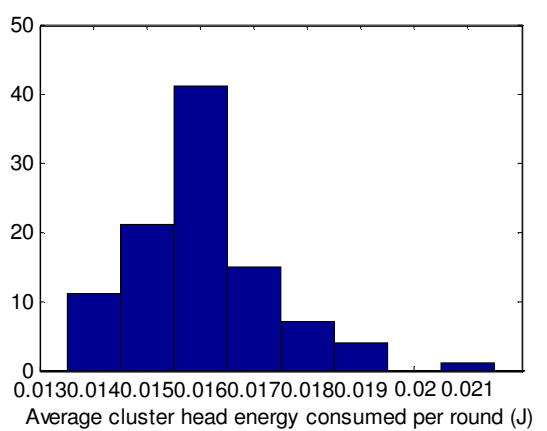

(a)

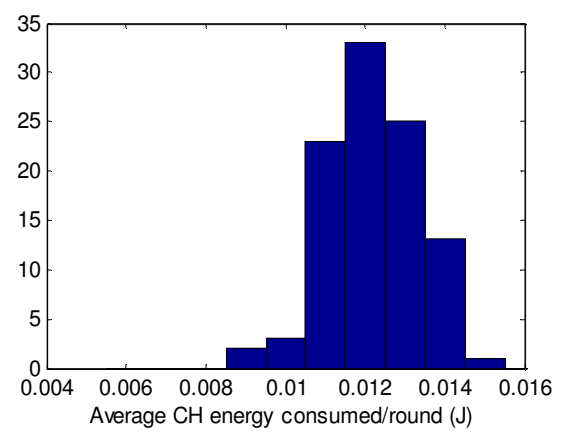

(c)

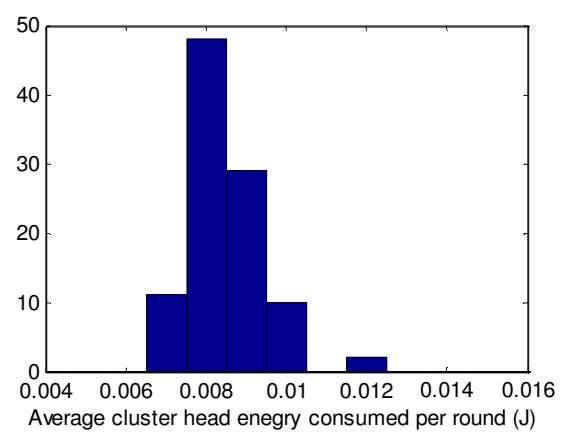

(b)

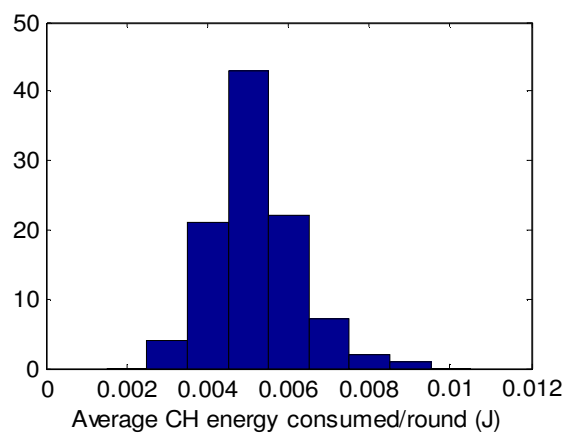

(d)

Figure 7: Histogram for average cluster head energy consumed per round (J). (a) 400 Nodes Direct communication case (b) 400 Nodes with mobile actors (c) 200 nodes with direct communication case (d) 200 Nodes with mobile actors.

\section{Conclusions and Future Work}

In this paper we have presented a simple and elegant technique to use mobile actor nodes for extending the network life time. We have shown that by deploying a small number of resource rich mobile nodes the network life time can enhance in excess of $100 \%$ as compared to single hop clustering. We presented a weighted cost based function that allows mobile actors to fine tune their location by considering the residual energy levels of associated cluster heads. We intend to investigate effects of different mobility models in our future research. 


\section{References}

[1] I. F. Akyildiz and I. H. Kasimoglu, "Wireless sensor and actor networks: research challenges," Ad Hoc Networks, vol. 2, pp. 351-367, 2004.

[2] W. Heinzelman, A. Chandrakasan, and H. Balakrishnan, " An Application-Specific Protocol Architecture for Wireless Microsensor Networks," IEEE Transactions on Wireless Communications, vol. 1, pp. 660-670, 2002.

[3] R. C. Shah, S. Roy, S. Jain, and W. Brunette, "Data MULEs: modeling and analysis of a three-tier architecture for sparse sensor networks," Ad Hoc Networks, vol. 1, pp. 215-233, 2003.

[4] Y. Gu, D. Bozdag, E. Ekici, F. Ozguner, and C. G. Lee, "Partitioning based mobile element scheduling in wireless sensor networks," Sensor and Ad Hoc Communications and Networks, 2005. IEEE SECON 2005. 2005 Second Annual IEEE Communications Society Conference on, pp. 386-395, 2005.

[5] W. Zhao and M. H. Ammar, "Message ferrying: proactive routing in highlypartitioned wireless ad hoc networks," Distributed Computing Systems, 2003. FTDCS 2003. Proceedings. The Ninth IEEE Workshop on Future Trends of, pp. 308-314, 2003.

[6] W. Zhao, M. Ammar, and E. Zegura, "A message ferrying approach for data delivery in sparse mobile ad hoc networks," Proceedings of the 5th ACM international symposium on Mobile ad hoc networking and computing, pp. 187-198, 2004.

[7] K. Dasgupta, K. Kalpakis, and P. Namjoshi, "An efficient clustering-based heuristic for data gathering and aggregation in sensor networks," Wireless Communications and Networking, 2003. WCNC 2003. 2003 IEEE, vol. 3, 2003.

[8] N. Aslam, W. Robertson, S. C. Sivakumar, and W. Phillips, "Energy Efficient Cluster Formation Using Multi-Criterion Optimization for Wireless Sensor Networks," in Proc. 4th IEEE Consumer Communications and Networking Conference (CCNC), 2007.

[9] M. Ye, C. F. Li, G. H. Chen, and J. Wu, "EECS: An Energy Efficient Clustering Scheme in Wireless Sensor Networks," IEEE Int'l Performance Computing and Communications Conference (IPCCC), pp. 535-540, 2005. 